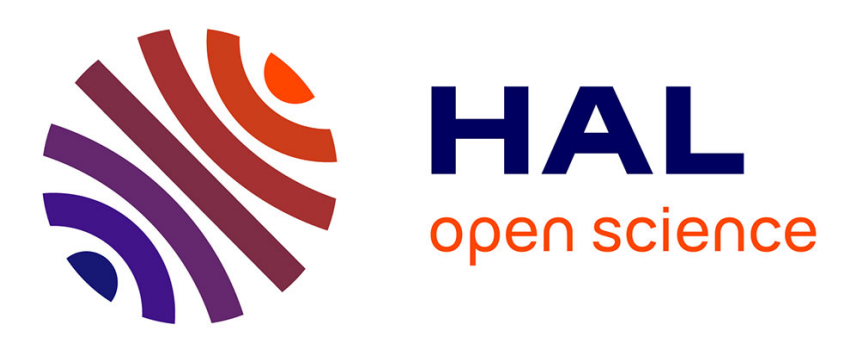

\title{
Segmentation of the cortex in fetal MRI using a topological model
}

Benoît Caldairou, Nicolas Passat, Piotr Habas, Colin Studholme, Mériam Koob, Jean-Louis Dietemann, François Rousseau

\section{To cite this version:}

Benoît Caldairou, Nicolas Passat, Piotr Habas, Colin Studholme, Mériam Koob, et al.. Segmentation of the cortex in fetal MRI using a topological model. International Symposium on Biomedical Imaging (ISBI), 2011, Chicago, United States. pp.2045-2048, 10.1109/ISBI.2011.5872814 • hal-01695037

\section{HAL Id: hal-01695037 \\ https://hal.univ-reims.fr/hal-01695037}

Submitted on 3 Feb 2018

HAL is a multi-disciplinary open access archive for the deposit and dissemination of scientific research documents, whether they are published or not. The documents may come from teaching and research institutions in France or abroad, or from public or private research centers.
L'archive ouverte pluridisciplinaire HAL, est destinée au dépôt et à la diffusion de documents scientifiques de niveau recherche, publiés ou non, émanant des établissements d'enseignement et de recherche français ou étrangers, des laboratoires publics ou privés. 


\title{
SEGMENTATION OF THE CORTEX IN FETAL MRI USING A TOPOLOGICAL MODEL
}

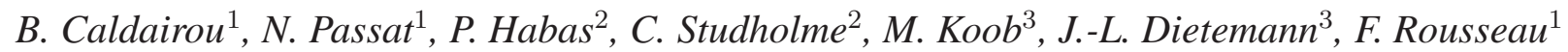 \\ ${ }^{1}$ LSIIT, UMR 7005 CNRS-Université de Strasbourg, France \\ ${ }^{2}$ BICG, University of California San Francisco, USA \\ ${ }^{3}$ LINC, FRE 3289 CNRS-Université de Strasbourg, France
}

\begin{abstract}
The study of in utero fetal MR images is essential for the diagnosis of abnormal brain development and the study of the maturation of the brain structures. However, few automated segmentation methods have been developed so far, compare to the numerous ones existing for the adult brain anatomy, which is due to the particular properties of these images. In this paper, we propose a two-step cortex segmentation technique including anatomical priors and a topological model. Experiments performed on in utero MR data and validation by comparison to experts segmentation emphasize the relevance of the method.
\end{abstract}

Index Terms - Cortex, fetal brain, segmentation, clustering, topology

\section{INTRODUCTION}

The study of in utero fetal brain is a key point for understanding the early development of brain structures and to propose new tools for diagnosis and follow the evolution of pathologies. A prerequisite is the automated labeling of these structures, which has to be robust to noise, fetal motion artefacts, partial volume effects (PVE), and magnetic resonance (MR) intensity inhomogeneity.

A first attempt for fetal brain structures segmentation was a semi-automated algorithm based on a region-growing method [1]. Later on, fully automatic techniques were developed. Bach Cuedra et al. [2] introduced separated Bayesian segmentation and Markov random field (MRF) regularization steps, the latter including anatomical priors. Other methods rely on motion-corrected $3 \mathrm{D}$ volumes, computed through reconstruction techniques of in utero MR scans [3, 4]. Habas et al. developed an automatic atlas-based segmentation [5], and a method including anatomical constraints in form of a laminar prior [6]. Finally, Gholipour et al. [7] performed a volumetric study of the brain based on the segmentation of the

The research leading to these results has received funding from the European Research Council under the European Community's Seventh Framework Programme (FP7/2007-2013 Grant Agreement no. 207667). This work is also funded by NIH Grant R01 NS055064 and a CNRS grant for collaboration between LSIIT and BICG. pericerebral fluid spaces (PFS) (the part of the cerebrospinal fluid (CSF) located around the cortical area) by using levelsets, connected components, and mathematical morphology filters.

Since the cortex segmentation is a prerequisite for cortical thickness measures or sulci study, a method to retrieve this structure based on intensity-based features and anatomical knowledge is presented. The cortex being a thin dark layer between the PFS and the white matter, the segmentation is achieved by considering the separation of the CSF into PFS and intraventricular fluid (ICSF). Moreover, a topological model [8] is introduced in order to deal with the partial volume effect by introducing geometrical constraints between the brain structures through the process.

\section{METHOD}

\subsection{Overview}

The fetal grey-level intracranial histogram presents two peaks corresponding respectively to the CSF and the brain. The analysis of a ground truth image histogram reveals an overlap between these two peaks, due mostly to the partial volume effect and the intensity inhomogeneity in the image (Fig. 1). Moreover, the cortex and the white matter are blended into the brain peak, meaning that these structures cannot be dissociated by using only image intensity.

The proposed method proceeds as follows (Fig. 2). First, a three class clustering of the intracranial volume, based on a topological model, is carried out in order to obtain the brain, the PSF and the ICSF. It allows us to estimate the border between the PFS and the brain. Since the cortex is located between the PFS and the white matter, this border is used to define a region of interest including the cortex. Finally, a three class clustering is performed to retrieve CSF, cortex and white matter.

\subsection{Local topological K-Means}

We define a topological model robust to intensity inhomogeneity, relying on three concentric spheres and introducing geometrical constraints for the segmentation process. 


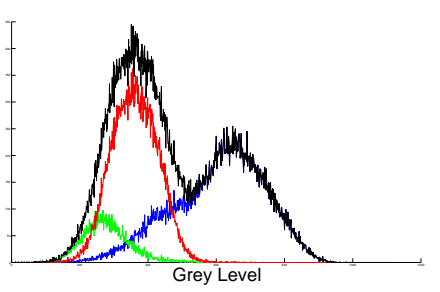

Fig. 1. Grey-level histogram from a fetal brain image. Black: intracranial volume, green: cortex, red: white matter and deep grey nuclei, blue: CSF.

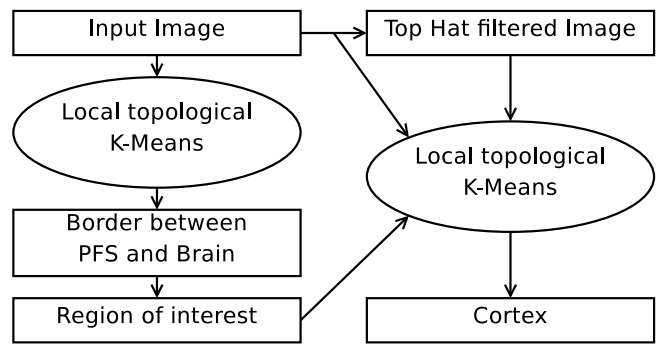

Fig. 2. Overall diagram of the segmentation process.

Let us consider an image composed of a set of voxels $\Omega$, each voxel $j \in \Omega$ having a given grey-level $\mathbf{y}_{j}$. Let us suppose that this image has to be segmented into $K(\geq 2)$ clusters. For each cluster $k$, let $S_{k}$ be the set of voxels included into it and $\nu_{k}$ be the centroid of this cluster (which usually corresponds to the mean grey-level value of this class of voxels). Based on these notations, in the $k$-means approach, the segmentation process of a grey-level image consists of the minimization of an energy function:

$$
J_{k-\text { means }}=\sum_{\mathbf{y}_{j} \in S_{k}} \sum_{k=0}^{K}\left\|\mathbf{y}_{j}-\nu_{k}\right\|_{2}^{2} .
$$

Nevertheless, considering a global centroid (therefore spatially invariant) makes the $k$-means algorithm sensitive to intensity inhomogeneity occurring in MRI data. In order to tackle this problem without relying on ad hoc prior knowledge related to the intensity inhomogeneity, we introduce local intensity centroid values $\nu_{j k}$. These local mean-values are computed in the following way. Let $\nu_{k}^{r}$ be the mean value of the $k^{t h}$ cluster in an image region $r$. This region mean value is considered as being located in the center of this region. Let $p_{r}$ be this position. Afterwards, for each considered voxel, a local mean-value $\nu_{j k}$ is computed by a distance-based interpolation of the nearest region mean-values: $\nu_{j k}=\frac{\sum_{r} \omega_{j r} \nu_{k}^{r}}{\sum_{r} \omega_{j r}}$, where $\omega_{j r}=1 / d\left(j, p_{r}\right)$ and $d\left(j, p_{r}\right)$ is the Euclidean spatial distance between the voxel $j$ and $p_{r}$.

The minimization of the $k$-means objective function is achieved by a border voxel exchange, with respect to the following topological model. Let $N_{j}$ be the neighborhood of voxel $j$. Let $C_{N_{j}}$ be the corresponding set of clusters present in $N_{j}$. A considered voxel $j$ switches from cluster $k$ to another candidate cluster $k^{\prime}$ if it meets the following requirements (Fig. 3):

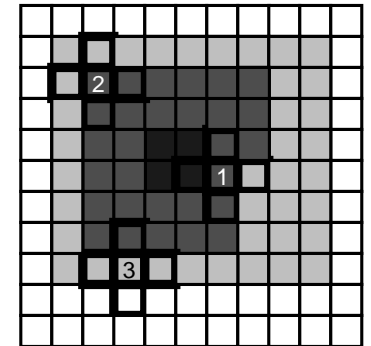

Fig. 3. Topological model. From white to dark grey, labels are 0,1 , 2 and 3, 0 being the background. Voxel 1: not eligible for switching to another label because there are three different labels in its neighborhood and a switch would break the concentric circle model. Voxel 2: eligible to switch to label 1. Voxel 3: not eligible to switch to label 2 because a neighbor is a background label.

$$
\left\{\begin{array}{l}
\left|C_{N_{j}}\right|=2, \\
\forall c \in C_{N_{j}}, c \neq \text { background, } \\
\left\|\mathbf{y}_{j}-\nu_{j k^{\prime}}\right\|_{2}<\left\|\mathbf{y}_{j}-\nu_{j k}\right\|_{2} .
\end{array}\right.
$$

In other words, a voxel is eligible for switching from one cluster to another if there is exactly two different clusters in its neighborhood, and if neither of these is the background. This formulation guarantees the invariance of the topological model by disallowing any circle break and preserving the background border.

\subsection{Tissue segmentation}

\subsection{1. $C S F$}

The segmentation is initialized by first computing an intracranial distance map (by using a chamfer distance) from the surface of the intracranial volume, which is obtained by a manual segmentation.

Then, we set the initial model by building three concentric spheres. The first sphere corresponds to the PFS and is a one voxel thin layer from the border of the intracranial volume. The second sphere represents the brain and the deepest one is set as ICSF.

Finally, we perform the segmentation in the way described in section 2.2 with the grey-level image as an input.

\subsubsection{Cortex}

Thanks to the PFS segmentation, it is now possible to build a region of interest where a 3 -class clustering can retrieve the cortex. Nevertheless, even in a reduced area, the cortex and the white matter can still not be dissociated on intensity-based features only.

The region of interest is built as stated next. From the border, we build a band including CSF and brain. This band is then divided in three sub-bands following the topological model. The CSF voxels are not changed and the other voxels are set as cortex and white matter.

Let $I: \Omega \rightarrow V$ be a discrete grey-level image. Let $\varphi_{B}$ be the morphological closing of $I$ by a structuring element $B$. 


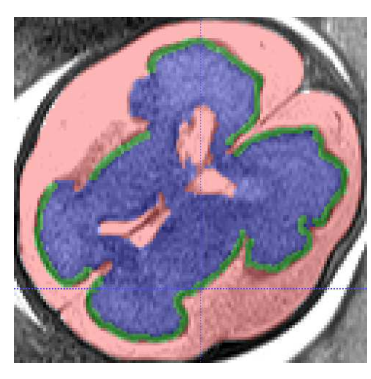

(a)

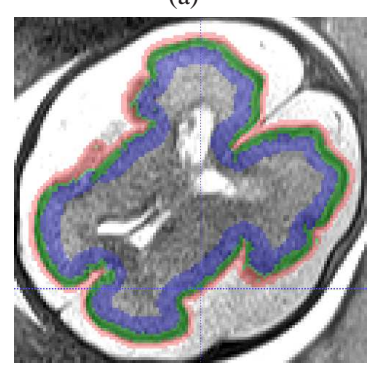

(d)

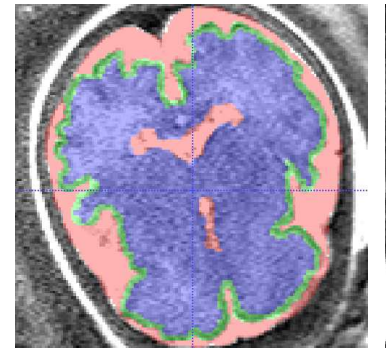

(b)

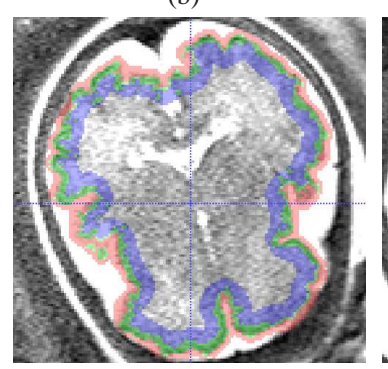

(e)

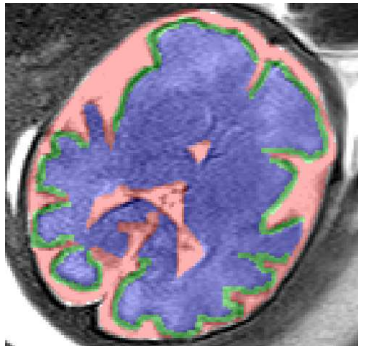

(c)

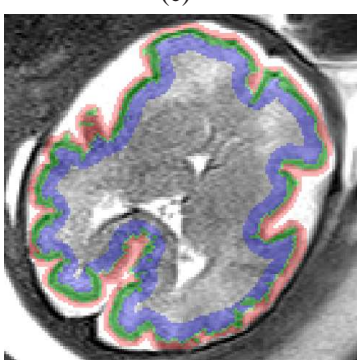

(f)

Fig. 4. Cortex extraction. (a, b, c): ground truth images, (d, e, f): region of interest with final segmentations. Red: CSF, green: cortex, blue: white matter and deep grey nuclei.

The Top Hat Dark Filter $T_{d}$ is defined as: $T_{d}(I)=\varphi_{B}(I)-I$. In other words, this filter reveals the small objects of the image that are removed by the closing, depending on the choice of the structural element [9]. Since the fetal cortex is a thin layer between PFS and white matter, we can build a corresponding structuring element in order to highlight the image areas where it will most likely appear.

Finally, we perform the segmentation by using a vector composed by the original image and the top-hat filtered image, instead of the original grey-level values alone, as the input of the topological segmentation presented in section 2.2. Consequently, each cluster is characterized by a centroid vector composed of its grey-level mean-value and its top-hatfiltered image mean value, allowing a better discrimination of the cortex.

\section{EXPERIMENTS AND RESULTS}

\subsection{Material and experimental settings}

Experiments are performed on a set of six T2-weighted MR images, acquired from a 1.5T scanner (Magnetom Avanto, Siemens, Germany Erlangen) using single shot fast spin echo sequences (TR $3190 \mathrm{~ms}$, TE 139-147 ms). Gestational ages (GA) range from 27 to 34 weeks. These images have the following dimensions: $512 \times 512 \times 20$ voxels and the voxels dimensions are: $0.7 \times 0.7 \times 3.45$ and $0.7 \times 0.7 \times 4.6$ millimeters. Since voxels are anisotropic, our topological model evolves on a 2-dimension basis, by the use of a 4-neighborhoods.

A non-local means denoising filtering [10] is performed as a preprocess in order to have a better reliability. This filter takes advantage of the redundancy present in images; broadly speaking, a small neighborhood around a voxel, called a patch, may match patches around other voxels within the same scene, defined by a research area around the current voxel, selecting the most accurate voxels to replace the current voxels value. The size of the research area is: $11 \times 11 \times 3$ and the size of the patches is: $3 \times 3 \times 1$.

The regions considered to compute the local mean values have the following dimensions: $32 \times 32 \times 1$. Empirically, we chose a $5 \times 5 \times 1$ structuring element to perform the top hat filter.

For the PFS segmentation, the model was initialized as follows. On the border with the background, a 1 voxel thin layer is set as PFS. Then, the voxels being less than 25 millimeters away from this border are set as brain and the remaining ones are set as ICSF. This guarantees that the ICSF initial cluster will not include any PFS voxels.

Regarding the cortex segmentation, the model was initialized as follows. The first two-millimeters layer is set as CSF, the next 5 as cortex and the last 2 as white matter. These values were chosen according to tissues anatomical characteristics.

The computation times are: about 5 seconds for the nonlocal mean filter, 5 minutes for the extraction of the CSF and finally, 2 minutes for the cortex segmentation.

\subsection{Validation}

Each image has one manual segmentation done by medical experts, and three of them were segmented twice. The validation consists of the computation of the sensitivity (Sen) and the dice similarity coefficient $(D S C)$ between the manual and the automated segmentation of the cortex. Let $T P$ be the amount of true positives (number of detected cortex voxels), FP the amount of false positives (number of voxels 


\begin{tabular}{|c|c|c|c|c|}
\hline Case (GA) & Sen & 3D $D S C$ & 2D Max $D S C$ & 2D $\overline{D S C}$ \\
\hline $1(30)$ & 0.571 & 0.629 & 0.746 & 0,689 \\
\hline $2(34)$ & 0.501 & 0.546 & 0.615 & 0,548 \\
\hline $3(32)$ & 0.738 & 0.664 & 0.738 & 0,711 \\
\hline $4(-)$ & 0.577 & 0.582 & 0.641 & 0,587 \\
\hline $5(28)$ & 0.697 & 0.652 & 0.778 & 0,685 \\
\hline $6(27)$ & 0.767 & 0.718 & 0.808 & 0,753 \\
\hline
\end{tabular}

Table 1. Sensitivity, global $D S C$, maximum $2 \mathrm{D} D S C$, mean $D S C$ on a selection of adjacent slices for the segmented cortex.

\begin{tabular}{|c|c|c|c|}
\hline Case (GA) & Exp1 vs Exp2 & Seg vs Exp1 & Seg vs Exp2 \\
\hline $1(30)$ & 0.611 & 0.629 & 0.570 \\
\hline $2(34)$ & 0.610 & 0.496 & 0.546 \\
\hline $3(32)$ & 0.610 & 0.664 & 0.593 \\
\hline
\end{tabular}

Table 2. $D S C$ for the segmented cortex. Comparison between expert 1 and expert 2 , between automated segmentation and expert 1 , between automated segmentation and expert 2.

incorrectly classified as cortex) and $F N$ the amount of false negatives (number of undetected cortex voxels). The sensitivity is given by: $\operatorname{Sen}=T P /(T P+F N)$ and the dice coefficient by: $D S C=2 \times T P /(2 \times T P+F N+F P)$.

Table 1 presents the $3 \mathrm{D}$ Sen and $D S C$ for each case. Moreover, because of the voxels anisotropy, a maximum 2D $D S C$ and a mean 2D DSC from a selection of six to eight adjacent slides located between the brainstem and the top of the head are also computed. The results illustrate a promising estimation of the cortex, which is emphasized by the better scores of the $2 \mathrm{D}$ means $D S C$ compare to the $3 \mathrm{D}$ one. Qualitatively, a visual insight of the segmentation (Fig. 4) underlines the accuracy of the method, even though a slight under-segmentation can be observed in some areas (Fig. 4(e) and 4(f)).

In order to have a complementary evaluation of the method, a comparison between several experts has been driven, on the three cases where two manual segmentations are available. For each case, the 3D $D S C$ between two experts and between each experts and the automated segmentation is presented (Table 2). The $D S C$ values similarity illustrates that if segmentation errors can partially explain the results, it may also come from the ground-truth quality. It also underlines the ability of the segmentation process to produce an evaluation of the cortex comparable to an expert's one.

\section{CONCLUSION}

A topological based clustering method has been proposed for the segmentation of the cortex in fetal brain MR images, which takes advantages of anatomical knowledges.

The validation performed on T2-weighted images tends to emphasize the relevance of the proposed methodology, in particular by quantitative and qualitative comparison to manual segmentations. It has been shown that the combination of anatomical knowledge, intensity-based features and a topo- logical model is able to retrieve the cortex.

Further work will focus on the improvement of the segmentation method and its validation on additional cases and on $3 \mathrm{D}$ reconstructed volumes.

\section{REFERENCES}

[1] I. Claude, J.-L. Daire, and G. Sebag, "Fetal brain MRI, segmentation and biometric analysis of the posterior fossa," IEEE Transactions on Biomedical Engineering, vol. 51, pp. 617-626, 2004.

[2] M. Bach Cuedra, M. Schaer, A. Andr, L. Guibaud, S. Eliez, and J.-Ph. Thiran, "Brain tissue segmentation of fetal MR images," in Image Analysis for the Developing Brain, Workshop in MICCAI, 2009.

[3] K. Kim, P.A. Habas, F. Rousseau, O.A. Glenn, A.J. Barkovich, and C. Studholme, "Intersection based motion correction of multislice MRI for 3-D in utero fetal brain image formation," Medical Imaging, IEEE Transactions on, vol. 29, pp. 146-158, 2010.

[4] F. Rousseau, O. Glenn, B. Iordanova, C. RodriguezCarranza, D. Vigneron, J. Barkovich, and C. Studholme, "Registration-based approach for reconstruction of high-resolution in utero fetal MR brain images," Academic Radiology, vol. 13, pp. 1072-1081, 2006.

[5] P.A. Habas, K. Kim, F. Rousseau, O.A. Glenn, A.J. Barkovich, and C. Studholme, "Atlas-based segmentation of developing tissues in the human brain with quantitative validation in young fetuses," Human Brain Mapping, vol. 31, pp. 1348-1358, 2010.

[6] P.A. Habas, K. Kim, D. Chandramohan, F. Rousseau, O.A. Glenn, and C. Studholme, "Statistical model of laminar structure for atlas-based segmentation of the fetal brain from in-utero MR images," in SPIE, 2009, vol. 7259, pp. 725917-725924.

[7] A. Gholipour, J. Estroff, C. Barnewolt, S. Connolly, and S. Warfield, "Fetal brain volumetry through MRI volumetric reconstruction and segmentation," International Journal of Computer Assisted Radiology and Surgery, 2010, http://dx.doi.org/10.1007/s11548-010-0512-x.

[8] D.L. Pham, P.-L. Bazin, and J.L. Prince, "Digital topology in brain imaging," Signal Processing Magazine, IEEE, vol. 27, pp. 51-59, 2010.

[9] J. Serra, Image Analysis and Mathematical Morphology: Theoretical Advances, Image Analysis and Mathematical Morphology. Academic Press, 1988.

[10] A. Buades, B. Coll, and J. M. Morel, "A review of image denoising algorithms, with a new one," Multiscale Modeling \& Simulation, vol. 4, pp. 490-530, 2005. 